\title{
Egressos da Residência de Medicina de Família e Comunidade em Minas Gerais
}

\author{
Graduates from a Family and Community \\ Medicine Residence Program in Minas Gerais
}

\author{
Fabrícia Vieira de Matos \\ Marília Borborema Rodrigues Cerqueira ${ }^{I}$ \\ Anderson Wesley Medeiros Silva ${ }^{I}$ \\ Júlia de Castro Vieira Veloso ${ }^{I}$ \\ Karla Verônica Alves de Morais ${ }^{I}$ \\ Antônio Prates Caldeira
}

\section{PALAVRAS-CHAVE}

- Estratégia Saúde da Família;

- Atenção Primária à Saúde;

- Internato e Residência;

- Educação Médica;

- Desenvolvimento de Pessoal.

\section{KEYWORDS}

- Family Health Strategy;

- Primary Health Care;

- Internship and Residency;

- Medical Education;

- Staff Development.

Recebido em: 19/03/2013

Reencaminhado em: 21/10/2013

Aprovado em: 19/01/2014

\section{RESUMO}

Este estudo objetivou investigar alguns aspectos do perfil profissional e a inserção no mercado de trabalho dos egressos médicos da Residência em Medicina de Família e Comunidade (RMFC) no norte de Minas Gerais. Trata-se de um estudo transversal, desenvolvido com aplicação de questionários enviados por meio de correio eletrônico a todos os egressos da RMFC na região nos últimos dez anos. Esses dados foram processados por meio de planilha eletrônica. A validação do questionário on-line foi realizada pelo teste de Kappa. Responderam ao instrumento 42 dos 51 médicos localizados. Verificou-se predominância do sexo masculino, a maioria está na faixa etária entre 30 e 34 anos, residindo e trabalhando na área de formação, na mesma região. O principal vínculo trabalhista é de contrato para prestação de serviço. Os entrevistados que informaram dificuldades no desempenho profissional relataram problemas relacionados à estrutura organizacional da instituição/empresa e necessidade de educação permanente. No mercado de trabalho do egresso da residência em Saúde da Família um dos grandes desafios encontrados é a precarização das relações de trabalho, algumas vezes, dependentes de oscilações políticas locais.

\section{ABSTRACT}

This study aimed to investigate some aspects of the professional profile and insertion into the labor market for graduates of Medical Residency in Family and Community Medicine (MRFMC) in the north of Minas Gerais, Brazil. This is a cross-sectional study developed with questionnaires that were sent via email to all graduates of MRFMC in the area over the last 10 years. These data were processed using a spreadsheet. The validation of the online questionnaire was conducted by the Kappa test. The questionnaire was answered by 42 of the 51 physicians located. There was a predominance of males, mostly aged between 30 and 34 years, living and working in the area of training, in the same region. Service agreements represent the main form of employment of these professionals. Respondents who reported difficulties in job performance cited problems related to the organizational structure of the institution/company and the need for continuous education. One of the major challenges faced in the labor market by Family Health Medical Residency graduates is the instability in their employment relations, sometimes dependent on local political movements. 


\section{INTRODUÇÃO}

O Sistema Único de Saúde (SUS), apesar de regulamentado desde 1990, tem sido descrito como um processo social em construção, já que as reformas sociais na democracia são, por natureza, lentas. Não há como negar os avanços registrados, sobretudo na ampliação do acesso aos serviços de saúde por parte da população mais carente ${ }^{1}$. Entretanto, ainda existem entraves ao pleno desenvolvimento do SUS que comprometem a operacionalização de seus princípios²

A formação e a qualificação de profissionais de saúde facilitam ou dificultam as mudanças para o fortalecimento do SUS, na medida em que criam sinergias com o novo modelo ou afastam o profissional dessa proposta de assistência à saúde. Alguns autores reconhecem os recursos humanos como capazes de gerar e conduzir processos transformadores no campo da saúde ${ }^{3,4}$. Além disso, o modelo assistencial proposto pelo SUS, com ênfase em uma atenção primária fortalecida, que preza a integralidade, a humanização e a promoção da saúde, é altamente dependente do perfil de formação e da prática dos profissionais de saúde $\mathrm{e}^{5,6}$.

No Brasil, a operacionalização da atenção primária é conduzida pela Estratégia Saúde da Família (ESF), que representa uma possibilidade de reorganização do modelo assistencial com ênfase na promoção da saúde. Essa nova proposta, entretanto, implica um grande desafio para a área de recursos humanos em saúde ${ }^{5,7}$.

Como as mudanças nos cursos de graduação para a formação de profissionais mais adequados para a assistência na ESF são lentas e heterogêneas ${ }^{8}$, parece necessária a formação no âmbito de pós-graduação para o exercício competente da prática profissional ${ }^{9}$. Dessa forma, a implantação de cursos de especialização nessa área da saúde, como estratégia de fortalecimento de políticas públicas, tem sido uma ação habitualmente utilizada pelo Ministério da Saúde (MS) ${ }^{5}$. Em Minas Gerais, o Hospital Universitário Clemente de Faria (HUCF), da Universidade Estadual de Montes Claros (Unimontes), ofertou o primeiro Curso de Especialização na Modalidade Residência em Saúde da Família, para médicos e enfermeiros em 1999. Em 2005, o componente médico do curso obteve credenciamento pela Comissão Nacional de Residência Médica (CNRM), com nova nomenclatura, de Residência em Medicina de Família e Comunidade ${ }^{10}$.

Entendendo a necessidade de avaliar os resultados e implicações da formação especializada para a atenção primária, este estudo teve como objetivo caracterizar alguns aspectos do perfil do egresso do Programa de Residência em Medicina de Família e Comunidade do HUCF/Unimontes, bem como avaliar sua inserção no mercado de trabalho.

\section{MÉTODO}

Trata-se de um estudo transversal, exploratório e descritivo, que representa um recorte dos dados referentes ao estudo sobre a Residência Multiprofissional em Saúde da Família no norte de Minas Gerais. A pesquisa foi conduzida com médicos egressos da residência, que responderam a um questionário disponibilizado no sítio eletrônico da Estação de Pesquisa da Unimontes durante os meses de fevereiro e março de 2011.

O acesso ao questionário se deu por meio de códigos identificadores enviados para o correio eletrônico de cada egresso. Foram enviados e-mails a todos os médicos que concluíram a residência de Medicina de Família e Comunidade até a data do envio e que disponibilizaram endereço eletrônico. A mensagem eletrônica, além do código identificador para acesso ao questionário, continha um texto que convidava o egresso a participar do estudo.

O instrumento de coleta de dados incluía entre suas variáveis: idade, gênero, estado civil, cidade de realização do curso, local de trabalho e moradia, atuação profissional, remuneração, dificuldades na atuação profissional e grau de influência do curso na prática profissional. Esses dados foram processados por meio de planilha eletrônica. A validação do questionário on-line foi realizada pelo teste de Kappa. Este teste avalia a concordância entre duas avaliações para além daquela que ocorre ao acaso. O objetivo dessa avaliação foi certificar a interpretação dos respondentes. O teste Kappa tem uma variação entre 0 e 1 , sendo considerados resultados satisfatórios aqueles mais elevados. Para utilização e análise do teste, foi necessário aplicar o questionário on-line por duas vezes em cerca de $10 \%$ dos egressos componentes da população entrevistada, em um período de 15 dias.

Este estudo foi aprovado pelo Comitê de Ética em Pesquisa da Unimontes (Parecer consubstanciado no 1.959/2010). O Termo de Consentimento Livre e Esclarecido foi disponibilizado on-line aos participantes para aceite eletrônico.

\section{RESULTADOS}

O Programa de Residência Médica estudado em Minas Gerais aconteceu nos municípios de Montes Claros, Santo Antônio do Amparo e Patos de Minas e produziu, entre 1999 e 2010, 53 médicos concluintes. Destes, 51 foram localizados por meio de endereço eletrônico e receberam o questionário, dos quais 42 o responderam, correspondendo a uma taxa de resposta de 82,3\%. Entre os não respondentes, dois não aceitaram participar do estudo e sete não acessaram o questionário eletrônico (informação identificada pelo sistema informático utilizado). 
A análise da validação realizada pelo teste Kappa mostrou uma concordância quase perfeita, variando de 0,80 a 1,00 para todas as questões. Desse modo, é improvável que os egressos tenham apresentado dificuldades na interpretação do questionário.

As principais características do grupo estudado são apresentadas na Tabela 1.

TABELA 1

Características dos egressos de uma residência de Medicina de Família e Comunidade de Minas Gerais; Montes Claros (MG), 2011

\begin{tabular}{|c|c|c|c|c|c|c|}
\hline \multirow{2}{*}{ Características } & \multicolumn{2}{|c|}{ Masculino } & \multicolumn{2}{|c|}{ Feminino } & \multicolumn{2}{|c|}{ Total } \\
\hline & (n) & $(\%)$ & (n) & $(\%)$ & (n) & $(\%)$ \\
\hline Sexo & 23 & 54,8 & 19 & 45,2 & 42 & 100 \\
\hline \multicolumn{7}{|l|}{ Faixa etária } \\
\hline Menos de 30 anos & 2 & 4,8 & 0 & 0 & 2 & 4,8 \\
\hline 30 a 39 anos & 17 & 40,5 & 15 & 35,7 & 32 & 76,2 \\
\hline 40 a 49 anos & 3 & 7,1 & 1 & 2,4 & 4 & 9,5 \\
\hline 50 a 59 anos & 1 & 2,4 & 3 & 7,1 & 4 & 9,5 \\
\hline \multicolumn{7}{|l|}{ Estado civil } \\
\hline Solteiro & 7 & 16,6 & 2 & 4,8 & 9 & 21,4 \\
\hline União estável/casado & 14 & 33,3 & 13 & 30,9 & 27 & 64,2 \\
\hline Separado/divorciado & 2 & 4,8 & 4 & 9,6 & 6 & 14,4 \\
\hline \multicolumn{7}{|c|}{ Local de moradia/trabalho } \\
\hline $\begin{array}{l}\text { Capital de MG e regiões } \\
\text { metropolitanas }\end{array}$ & 4 & 9,6 & 0 & 0 & 4 & 9,6 \\
\hline Norte de MG & 13 & 30,9 & 15 & 35,7 & 28 & 66,6 \\
\hline Outras regiões de $\mathrm{MG}$ & 4 & 9,6 & 3 & 7,1 & 7 & 16,7 \\
\hline Outros Estados & 2 & 4,8 & 1 & 2,4 & 3 & 7,1 \\
\hline \multicolumn{7}{|c|}{ Faixa de renda mensal em salários mínimos* } \\
\hline$<10$ salários & 2 & 4,8 & 2 & 4,8 & 4 & 9,6 \\
\hline 10 a 15 salários & 7 & 16,8 & 11 & 26,4 & 18 & 43,2 \\
\hline Mais que 15 salários & 13 & 30,9 & 2 & 4,8 & 15 & 35,7 \\
\hline Não declarado & 3 & 7,2 & 2 & 4,8 & 5 & 11,9 \\
\hline \multicolumn{7}{|c|}{ Aumento da renda após o curso } \\
\hline $\operatorname{Sim}$ & 14 & 33,3 & 13 & 31,0 & 27 & 64,3 \\
\hline Não & 9 & 21,4 & 6 & 14,3 & 15 & 35,7 \\
\hline
\end{tabular}

* Valor do salário mínimo no período de coleta de dados: $R$ \$ 540,00.

Em relação ao gênero, 54,8\% dos egressos $(n=23)$ eram homens. A maioria dos médicos $(76,2 \% ; n=32)$ estava na faixa etária entre 30 e 39 anos. Quanto ao estado civil, 64,2\% ( $n=27)$ declararam-se casados ou em união estável. Em relação ao lo- cal de trabalho e residência, $66,6 \%(n=28)$ moram e trabalham no norte de Minas Gerais.

A maioria dos egressos do gênero masculino (30,9\%; $\mathrm{n}=$ 13) relatou possuir renda maior que 15 salários mínimos, enquanto a maioria das médicas egressas $(26,4 \%$; $n=11)$ informou possuir renda entre 10 e 15 salários mínimos. $\mathrm{O}$ aumento da renda após a conclusão do curso de residência médica foi relatado por $64,3 \%$ dos egressos $(n=27)$. Ressalta-se que os entrevistados, enquanto estavam realizando o curso de residência pesquisado, em Montes Claros (MG), recebiam a bolsa nacional de residência médica e uma complementação financeira por parte da Prefeitura Municipal de Montes Claros.

TABELA 2

Tipo de trabalho de egressos médicos, por sexo dos egressos do curso de Residência em Medicina de Família e Comunidade; Montes Claros (MG), 2011

\begin{tabular}{|c|c|c|c|c|c|c|}
\hline \multirow{3}{*}{ Tipo de trabalho } & \multicolumn{4}{|c|}{ Sexo } & \multirow{2}{*}{\multicolumn{2}{|c|}{ Total }} \\
\hline & \multicolumn{2}{|c|}{ Feminino } & \multicolumn{2}{|c|}{ Masculino } & & \\
\hline & (n) & $(\%)$ & (n) & $(\%)$ & (n) & $(\%)$ \\
\hline \multicolumn{7}{|l|}{ Assistência } \\
\hline APS, integrante de ESF & 13 & 30,9 & 18 & 42,8 & 31 & 73,8 \\
\hline $\begin{array}{l}\text { APS, não integrante de } \\
\text { ESF }\end{array}$ & 1 & 2,4 & 3 & 7,1 & 4 & 9,5 \\
\hline $\begin{array}{l}\text { Atenção secundária } \\
\text { pública }\end{array}$ & 4 & 9,5 & 4 & 9,5 & 8 & 19,0 \\
\hline Hospitalar pública & 2 & 4,8 & 4 & 4 & 6 & 14,2 \\
\hline Hospitalar privada & 0 & 0 & 6 & 14,2 & 6 & 14,2 \\
\hline Outro serviço privado & 5 & 11,9 & 5 & 11,9 & 10 & 23,8 \\
\hline \multicolumn{7}{|l|}{ Gestão } \\
\hline $\begin{array}{l}\text { Coordenação ou gerência } \\
\text { em Saúde da Família }\end{array}$ & 1 & 2,4 & 2 & 4,8 & 3 & 7,1 \\
\hline Instituição hospitalar & 0 & 0 & 1 & 2,4 & 1 & 2,4 \\
\hline \multicolumn{7}{|l|}{ Docência } \\
\hline Instituição pública & 9 & 21,4 & 7 & 16,7 & 16 & 38,0 \\
\hline Instituição particular & 9 & 21,4 & 7 & 16,7 & 16 & 38,0 \\
\hline \multicolumn{7}{|l|}{ Pesquisa } \\
\hline Instituição pública & 1 & 2,4 & 1 & 2,4 & 2 & 4,8 \\
\hline Outra atividade & 1 & 2,4 & 6 & 14,2 & 7 & 16,6 \\
\hline Não informado & 1 & 2,4 & 0 & 0 & 0 & 2,4 \\
\hline
\end{tabular}

Nota: Neste quesito, os entrevistados responderam a mais de uma alternativa, e o total de respostas foi 116 . A \% total se refere ao número de entrevistados.

A Tabela 2 apresenta a distribuição dos egressos da Residência de Medicina de Família e Comunidade segundo o tipo de trabalho atual. Observou-se que 73,8\% (n = 31) dos egressos trabalham na assistência à saúde, em equipes da ESF. Vários egressos informaram atuar também em outras atividades. Foram registradas as seguintes áreas de atuação, além da 
assistência: gestão de serviços de saúde $(9,5 \%$; $n=4)$, docência $(38,0 \% ; n=16)$ e pesquisa $(4,8 \% ; n=2)$.

Foi investigado o vínculo de trabalho com a maior carga horária e o vínculo com a maior remuneração (Tabela 3). Dos entrevistados, 59,5\% ( $\mathrm{n}=25)$ estavam vinculados por meio de contratos de prestação de serviço por tempo determinado, em relação tanto à instituição onde possuíam maior carga horária, como à instituição onde recebiam maior remuneração. Outros vínculos registrados mais destacados foram: regime estatutário e celetista.

\section{TABELA 3}

Vínculo trabalhista dos egressos da residência de Medicina de Família e Comunidade em relação

à carga horária e remuneração no trabalho; Montes Claros (MG), 2011

\begin{tabular}{lcccc}
\hline \multirow{2}{*}{ Tipo de vínculo } & \multicolumn{4}{c}{ Tipo de instituição } \\
\cline { 2 - 5 } & Maior carga horária & Maior remuneração \\
\cline { 2 - 5 } & (n) & $\mathbf{( \% )}$ & (n) & $\mathbf{( \% )}$ \\
\hline Cooperado & 1 & 2,4 & 1 & 2,4 \\
Pessoa jurídica & 1 & 2,4 & 1 & 2,4 \\
Carteira assinada & 7 & 16,7 & 0 & 07 \\
Regime estatuário & 2 & 4,8 & 8 & 19,2 \\
Autônomo & 3 & 7,1 & 4 & 9,6 \\
Contratado & 25 & 59,5 & 25 & 59,5 \\
Outro & 2 & 4,8 & 2 & 4,8 \\
Não informado & 1 & 2,4 & 1 & 2,4 \\
Total & 42 & 100 & 42 & 100 \\
\hline
\end{tabular}

Nota: Empregado com carteira assinada é regime CLT por prazo indeterminado; regime estatuário é regido pelo estatuto do servidor público federal, ou estadual, ou municipal; contratado é o trabalhador empregado regido pela CLT, porém com prazo determinado; autônomo é a pessoa física contratada por prestação de serviço.

Ao serem questionados sobre os benefícios da conclusão da residência estudada (Tabela 4), 88,1\% dos egressos $(n=37)$ destacaram a melhoria no desempenho profissional. A percepção subjetiva dos egressos em relação à sua formação também foi aferida, sendo que $97,6 \%(n=41)$ dos respondentes destacaram a residência como "importante" ou "muito importante". O nível de satisfação com o curso foi classificado como "excelente" ou "ótimo" por 50,0\% dos egressos (n = 21). O grau de influência do curso na prática profissional foi expresso como "muito influenciada" ou "totalmente influenciada" por $83,3 \%$ dos egressos $(n=35)$.
TABELA 4

Percepções dos egressos da residência em Medicina de Família e Comunidade sobre o processo de formação; Montes Claros (MG), 2011

\begin{tabular}{|c|c|c|}
\hline \multirow{2}{*}{ Características } & \multicolumn{2}{|c|}{ Respostas } \\
\hline & $\mathbf{n}$ & $\%$ \\
\hline \multicolumn{3}{|c|}{ Benefícios de conclusão do curso de residência pesquisado* } \\
\hline Novos conhecimentos sobre a profissão & 34 & 80,9 \\
\hline Melhoria no desempenho profissional & 37 & 88,1 \\
\hline Realização profissional/pessoal & 23 & 54,8 \\
\hline Aumento de chance no mercado de trabalho & 19 & 45,2 \\
\hline Mudança de paradigmas pessoais e profissionais & 26 & 61,9 \\
\hline Possibilidade de melhoria salarial & 19 & 45,2 \\
\hline Promoção de função/cargo & 7 & 16,7 \\
\hline Outros & 9 & 21,4 \\
\hline Não houve benefícios & 1 & 2,4 \\
\hline \multicolumn{3}{|l|}{ Grau de importância atribuído à residência } \\
\hline Muito importante & 26 & 61,9 \\
\hline Importante & 15 & 35,7 \\
\hline Pouco importante & 1 & 2,4 \\
\hline \multicolumn{3}{|l|}{ Nível de satisfação com a residência } \\
\hline Excelente & 7 & 16,7 \\
\hline Ótimo & 14 & 33,3 \\
\hline Bom & 15 & 35,7 \\
\hline Regular & 6 & 14,3 \\
\hline \multicolumn{3}{|l|}{ Grau de influência da residência na prática profissional } \\
\hline Totalmente influenciada & 13 & 30,9 \\
\hline Muito influenciada & 22 & 52,4 \\
\hline Pouco influenciada & 5 & 11,9 \\
\hline Não é influenciada & 2 & 4,8 \\
\hline
\end{tabular}

(*) Neste quesito, os entrevistados responderam a mais de uma alternativa. Os percentuais referem-se à proporção dos respondentes em relação ao número total de entrevistados.

\section{DISCUSSÃO}

O presente estudo possibilitou a caracterização e o estudo da inserção profissional dos médicos egressos da Residência em Medicina de Família e Comunidade, revelando aspectos ainda não apontados pela literatura nacional sobre esses profissionais. O perfil identificado destaca um profissional diferenciado em relação ao padrão apontado em outros es- 
tudos $^{11,12}$. Neste estudo, o grupo de médicos se mostrou com idade ligeiramente superior, permitindo inferir que possuem maior maturidade e maior fixação na área da atenção primária. Outros estudos já mostraram que profissionais recém-formados e sem especialização tendem a apresentar maior instabilidade nas equipes de Saúde da Família ${ }^{13,14}$. Isso implica uma elevada rotatividade, o que representa uma questão crítica para o processo de gestão dos recursos humanos na área da saúde ${ }^{3}$.

A maior inserção dos profissionais estudados em equipes da ESF também aponta maior vinculação dos mesmos com a atenção primária, sendo também notória a fixação dos profissionais na região onde fizeram a residência de Medicina de Família e Comunidade (norte de Minas Gerais).

A renda mensal da maioria dos egressos esteve entre 10 e 15 salários mínimos, destacando-se um diferencial entre os gêneros, com maior percentual de profissionais masculinos recebendo mais de 15 salários mínimos. Este resultado é concordante com estudo apresentado pelo Conselho Regional de Medicina do Estado de São Paulo ${ }^{15}$, que revelou menor renda entre profissionais do gênero feminino. Curiosamente, as diferenças de renda por gênero são significativas principalmente quando se trata de trabalho que exige nível superior de escolaridade. A segregação das mulheres em ocupações de menor remuneração está presente em todas as áreas, mas, para ocupações que exigem nível superior de escolaridade, as diferenças de rendimento são ainda maiores ${ }^{16}$. No presente estudo, não foi possível apurar se as diferenças observadas são no mesmo município ou se decorrem de contratações em municípios distintos.

Um percentual não desprezível de egressos de programas de residência informou trabalhar em áreas distintas da sua especialização. Feuerwerker atribui esse fato à falta de ordenação entre a formação de especialistas e as necessidades do mercado ${ }^{17}$. Esse resultado também pode sugerir algum nível de insegurança com o trabalho, hipótese que é reforçada quando se analisa o tipo de vínculo dos profissionais avaliados. A grande maioria dos pesquisados informou vínculo precário, de contratação por tempo determinado. Essa precarização do vínculo e da qualidade do emprego na ESF já foi identificada pelo próprio Ministério da Saúde ${ }^{18}$. Também o estudo de Tomasi e colaboradores, realizado com trabalhadores da atenção básica nas regiões Nordeste e Sul do País, constatou que grande parte dos entrevistados tinha vínculo de trabalho tipicamente precário, sem garantias trabalhistas ${ }^{19}$.

As percepções dos egressos sobre o processo de formação profissional são bastante positivas, destacando o importante papel da residência médica para a formação diferenciada na área da atenção primária, em consonância com outro estudo ${ }^{20}$. Em outros países já foi apontado um crescimento da residência médica voltada para a medicina familiar e comunitária como estratégia que permite aos seus especialistas realizar a assistência mais adequada no contexto dos cuidados primários ${ }^{21}$.

Um estudo que analisou a trajetória profissional de egressos de cursos de especialização em Saúde da Família na região metropolitana de São Paulo observou também percepção positiva por parte dos profissionais, que apontaram que o curso superou as expectativas previstas, permitindo efetividade e organização do trabalho, além de melhor atendimento e estudo das famílias, resolução de problemas nas Unidades Básicas de Saúde e boa aplicação do curso em sua prática rotineira ${ }^{22}$. Por outro lado, esse mesmo estudo observou uma baixa identidade dos egressos com a ESF, com abandono das atividades da atenção primária. Pode-se inferir que os cursos de especialização não têm, portanto, o mesmo potencial dos cursos de residência.

Parece haver consenso sobre o fato de que a residência em Medicina de Família e Comunidade é a modalidade de formação mais adequada e de excelência para preparar o médico mais adequado ao contexto da $\mathrm{EFS}^{20}$. Leão e Caldeira registraram que equipes de ESF que contam com profissionais egressos de residência na área de Saúde da Família apresentam melhores resultados quando comparados com equipes sem egressos de cursos de residência9 .

Apesar da reconhecida relevância, dos resultados observados e do aumento substancial de programas e vagas para residência em Medicina de Família e Comunidade que têm sido registrados em todo o País nos últimos anos, menos da metade dessas vagas são ocupadas ${ }^{23}$. Trata-se de uma questão complexa que envolve desde o processo de graduação médica até as condições de trabalho e perspectivas de crescimento profissional, mas a literatura brasileira apresenta poucos estudos que abordam tais variáveis ${ }^{24}$. Infelizmente, essa situação não é uma particularidade nacional, e outros países também registram a dificuldade de formação de médicos de família. Alguns autores discutem a falta de modelos na área da atenção primária, reforçando a necessidade de reestruturação dos cursos de graduação para estimular e promover a formação de médicos com perfis mais adequados à medicina de família ${ }^{25,26}$.

\section{CONCLUSÃO}

Em síntese, este estudo identificou alguns aspectos do perfil dos médicos egressos da residência em Medicina de Família e Comunidade que podem auxiliar a formulação de políticas de formação de recursos humanos para a ESF. Apesar das limitações deste estudo, com amostra limitada a uma região específica, os resultados - que destacam a continuidade com 
a assistência à saúde em equipes da ESF, o aumento nos rendimentos após o curso, maiores facilidades no desempenho profissional e maior satisfação profissional - apontam aspectos que não devem ser negligenciados na formulação de propostas para aumentar a efetividade da ESF. Entende-se que o desejável fortalecimento da atenção primária passa por uma política mais efetiva de formação de recursos humanos, desde a graduação, sem prejuízos às práticas de pós-graduação e educação permanente.

\section{REFERÊNCIAS}

1. Paim J, Travassos C, Almeida C, Bahia L, Macinko J. The Brazilian health system: history, advances, and challenges. Lancet. 2011; 377(9779):1778-97.

2. Victora CG, Barreto ML, Carmo LM, Monteiro CA, Schmidt MI, Paim J, et al. Lancet Brazil Series Working Group. Health conditions and health-policy innovations in Brazil: the way forward. Lancet. 2011; 377(9782):2042-53.

3. Scalco SV, Lacerda JT Calvo MCM. Modelo para avaliação da gestão de recursos humanos em saúde. Cad. Saúde Pública. 2010; 26(3):603-614.

4. Maeda ST, Moleiro PF, Egry EY, Ciosak SI. Recursos humanos na Atenção Básica: investimento e força propulsora de produção. Rev Esc Enferm USP. 2011;45(Esp. 2):1651-5

5. Gil CRR. Formação de Recursos Humanos em Saúde da Família: paradoxos e perspectivas. Cad. Saúde Pública. $2005 ; 21(2): 490-8$.

6. Moretti-Pires RO. Complexidade em Saúde da Família e formação do futuro profissional de saúde. Interface: comunicação, saúde e educação. 2009; 13(30):153-66.

7. Haddad AE, Morita MC, Pierantoni CR, Brenelli SL, Passarella T, Campos FE. Formação de profi ssionais de saúde no Brasil: uma análise no período de 1991 a 2008. Rev. Saúde Pública. 2010; 44(3):383-93.

8. Costa RKS, Miranda FAN. Formação profissional no SUS: oportunidades de mudanças na perspectiva da estratégia de saúde da família. Trab Educ Saúde. 2008; 6(3):503-17.

9. Leão CDA, Caldeira AP. Avaliação da associação entre qualificação de médicos e enfermeiros em atenção primária em saúde e qualidade da atenção. Ciênc. saúde coletiva. 2011;16(11):4415-23.

10. Silvério JB. Residência Multiprofissional em Saúde da Família: o caso do Município de Montes Claros. In: Brasil. Ministério da Saúde. Secretaria de Gestão do Trabalho e da Educação na Saúde. Departamento de Gestão da Educação na Saúde. Residência multiprofissional em saúde: experiências, avanços e desafios. Brasília: Ministério da Saúde; 2006. 414 p.
11. Espíndola PS, Lemos CLS, Reis LBM. Perfil do profissional de nível superior na estratégia saúde da família. Rev Bras Promoç Saúde. 2011; 24(4):367-75.

12. Miranzi SSC, Mendes CA, Nunes AA, Iwamoto HH, Miranzi MAS, Tavares DMS. Qualidade de vida e perfil sociodemográfico de médicos da estratégia de saúde da família. Rev Med Minas Gerais 2010; 20(2):189-97.

13. Medeiros CRG, Junqueira AGW, Schwingel G, Carreno I, Jungles LAP, Saldanha OMFL. A rotatividade de enfermeiros e médicos: um impasse na implementação da Estratégia de Saúde da Família. Ciênc. saúde coletiva. 2010;15(Supl. 1):1521-31.

14. Campos CVA, Malik AM. Satisfação no trabalho e rotatividade dos médicos do Programa de Saúde da Família. RAP. 2008; 42(2):347-68.

15. Conselho Regional de Medicina do Estado de São Paulo. Perfil do médico [online]. São Paulo: Cremesp; 2007. [Capturado em 18 out. 2010]. Disponível em: http:/ / www.cremesp. org.br/library/modulos/sala_imprensa/arquivos / perfil_medico.pdf

16. Leone ET, Baltar P. A mulher na recuperação recente do mercado de trabalho brasileiro. Rev. bras. estud. popul. 2008. [Capturado em 28 Aug. 2012]. 25(2). Disponível em: $<$ http:/ / www.scielo.br/scielo.php?script=sci_arttext\&pi $\mathrm{d}=$ S010230982008000200003\&lng=en\&nrm=iso $>$.

17. Feuerwerker LCM. Changes in medical education and medical residency in Brazil. Interface (Botucatu) 1998; 2(3).

18. Brasil. Ministério da Saúde. Departamento de Gestão e da Regulação do Trabalho em Saúde/DEGERTS. Secretaria de Gestão do Trabalho e da Educação na Saúde/SGTES. Precarização e Qualidade do Emprego no Programa de Saúde da Família. Belo Horizonte (MG); 2007.

19. Tomasi E, Facchini LA, Piccini RX, Thumé E, Silveira DS, Siqueira FV, et al. Perfil sócio-demográfico e epidemiológico dos trabalhadores da atenção básica à saúde nas regiões Sul e Nordeste do Brasil. Cad. Saúde Pública. 2008; 24 Sup 1:S193-S201.

20. Castro VS, Nóbrega-Therrien SM. Residência de Medicina de Família e Comunidade: uma Estratégia de Qualificação. Rev. bras. educ. med. 2009; 33(2):211-20.

21. Suárez-Bustamante MA. La medicina familiar en el perú: evolución y desafíosRev Peru Med Exp Salud Publica. 2008; 25(3):309-15.

22. Silva TAM, Fracolli LA, Chiesa AM. Trajetória profissional na Estratégia Saúde da Família: em foco a contribuição dos cursos de especialização. Rev. Latino-Am. Enfermagem. 2011;19(1):[08 telas] 
23. Mello GA, Mattos ATR, Souto BGA, Fontanella BJB, Demarzo MMP. Médico de família: ser ou não ser? Dilemas envolvidos na escolha desta carreira. Rev. bras. educ. med. 2009; 33 (3):475-482.

24. Vasconcelos FGA, Zaniboni MRG. Dificuldades do trabalho médico no PSF. Ciência \& Saúde Coletiva. 2011; 16(Supl. 1):1494-1504.

25. Bethune C, Hansen PA, Deacon D, Hurley K, Kirby A, Godwin M. Family medicine as a career option: How students' attitudes changed during medical school. Can Fam Physician. 2007;53:880-885.

26. Brown JB, French R, McCulloch A, Clendinning E. Primary health care models: Medical students' knowledge and perceptions. Can Fam Physician. 2012;58:166-71

\section{CONTRIBUIÇÃO DOS AUTORES}

Fabrícia Vieira de Matos foi responsável pela orientação das atividades desenvolvidas, por analisar e interpretar os dados, revisão de literatura, elaboração e revisão final do artigo. Ma- rília Borborema Rodrigues Cerqueira foi responsável por analisar e interpretar os dados, revisão de literatura e elaboração do artigo. Anderson Wesley Medeiros Silva foi responsável pela coleta de dados e revisão da literatura. Júlia de Castro Vieira Veloso foi responsável pela coleta de dados e revisão da literatura. Karla Verônica Alves de Morais foi responsável pela coleta de dados e revisão da literatura. Antônio Prates Caldeira foi responsável por analisar e interpretar os dados, revisão de literatura, elaboração e revisão final do artigo.

\section{CONFLITO DE INTERESSES}

Declarou não haver

\section{ENDEREÇO PARA CORRESPONDÊNCIA}

Fabrícia Vieira de Matos

Rua João Pinheiro, 128 - apto 101

Centro - Montes Claros

CEP 38400-093 - MG

E-mail: fabricia.matos@unimontes.br 